\title{
Numerical Simulation on a Gas Distributor Used In Slurry Column Reactor
}

\author{
Yating CAI \\ College of Chemical Engineering, Beijing University of Chemical Technology, Beijing, 100029, \\ China \\ Email: caiyating_shirley@163.com
}

Keywords: CFD; Gas distributor; Slurry column reactor; Multiphase flow; Reflux

\begin{abstract}
The slurry column reactor has the most promising prospect in FT synthesis. In this paper, the gas distributor developed for slurry column rector was analyzed to find an improved gas distributor with small energy consumption, good distribution performance and the good characteristics of preventing backflow. A novel gas distributor which is employed in the slurry column reactor was proposed and multiphase flow patterns were numerically studied. The single phase flow patterns of the new slurry column gas distributor were simulated and the smaller preventing-backflow ball was found out. When connect the upper and lower two openings to form one opening, there is lower pressure drop in the gas distributor, unevenness also reduced accordingly. The overall gas holdup was consistent with the experimental data. The performance of the preventing-backflow ball was also studied in this paper. Some design requirements were put forward in order to stroke the liquid flow back.
\end{abstract}

\section{Introduction}

As a key equipment and internals in a slurry bubble column reactor, gas distributor has a great influence on the initial gas distribution and flow pattern and also promotes the stable operation of the entire bed. Gas distributor is located at the bottom of the slurry bed, whose main function is supporting the catalyst on the slurry column bed, uniformly distributing the gas, and inhibiting the development of aggregative fluidization and maintaining stability of the bed. At present engineers always take the perspective of uniform distribution into consideration to design gas distributor in slurry column reactor, while someone are beginning to consider the deposition of catalysts [1]. But less research focus on the description and analysis of the reflux causing by suddenly cutting off the gas source. With the continuous development of computer technology, CFD Simulations become a recognized and important research method [2] [3] [4]. Krishna [5], Dhotre and Smith [6] had already modeled the slurry column reactor by multiphase flow model and studied its amplification character. Delnoij [7] [8] modeled a large reactor by taking bubbles as discrete phase and slurry as the continuous phase, and the break and gathering of bubbles were taken into account. This paper based on numerical simulation of the three dimensional model, systematically analysis the gas flow field distribution inside and the influence of the bubble for the flow outside the gas distributor. Various parameters on the performance of the general rule were obtained, and provide basic theory and optimization ideas for designing blister distributor.

\section{Experiment Details}

Fig.1. shows the schematic of the experimental apparatus. These experiments were carried out in plexiglass columns. The distributors were also made of plexiglass, which was convenient for us to monitor experimental phenomenon and operational stability clearly. The experimental working medium was air and water. The compressed air was blown into the gas distributor layer through the buffer tank, the flowmeter and the solenoid valve, and then flowed out of the bottom of the column and into the surrounding environment. Air flow rate was measured by the rotameter, pressure drop were measured by $U$ tube. All experiments were carried out at ambient temperature and atmospheric pressure. The inlet flow rate of the monomer was $20,32,56,80,100 \mathrm{~m}^{3} / \mathrm{h}$ respectively. The 
experiment was carried out in a column with a diameter of $400 \mathrm{~mm}$. The experimental results obtained by Bed Expansion Method could be used to evaluate the overall gas holdup and prove the validity of the numerical model.

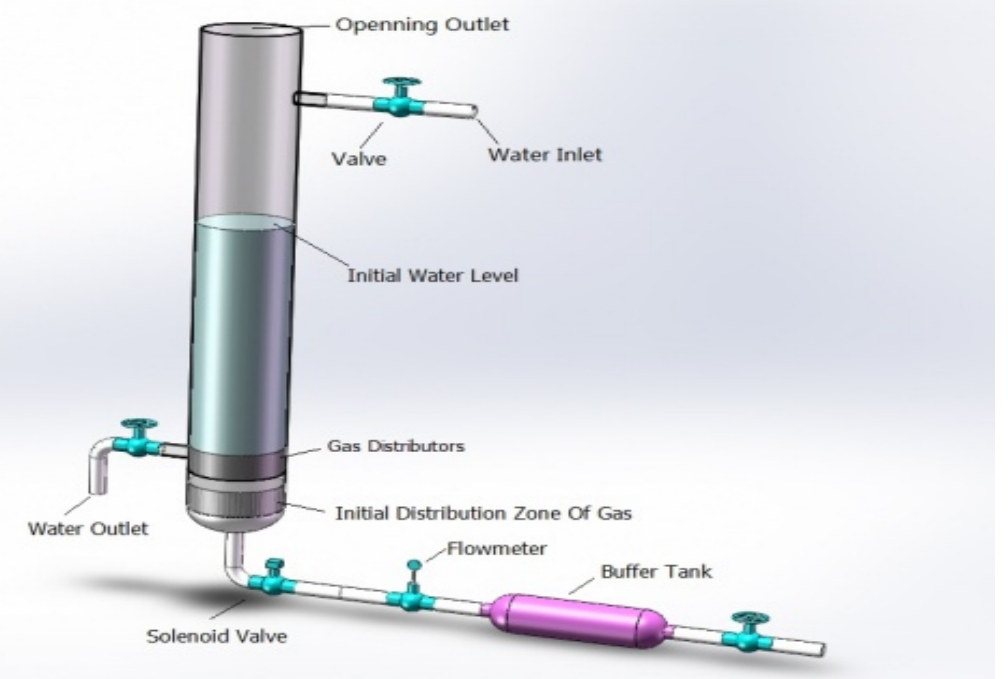

Fig.1. The schematic of the experimental system

\section{Numerical Models}

The nature of the fluid has a great influence in turbulent form. Therefore the CFX software introduced a number of turbulence models to meet the calculation of turbulent flow. In this paper, this model is $\mathrm{k}-\varepsilon$ model. The model is most frequently used in the simulation turbulence model, which was found a best balance point in its accuracy and the mathematical equations.

Continuity Equation: $\frac{\partial \rho}{\partial t}+\nabla \cdot(\rho U)=0$

Momentum equation: $\frac{\partial \rho U}{\partial t}+\nabla \cdot(\rho U \otimes U)-\nabla \cdot\left(\mu_{\text {eff }} \nabla U\right)=\nabla \cdot p^{\prime}+\nabla \cdot\left(\mu_{e f f} \nabla U\right)^{T}+B$

Where $B$ is total volume force; $\mu_{\text {eff }}$ is effective viscosity; $p$ ' is correction pressure.

$K, \varepsilon$ values can be solved directly from the turbulent kinetic energy and turbulent kinetic energy dissipation equation, turbulent kinetic energy equation is as follows:

$$
\begin{aligned}
& \frac{\partial(\rho U)}{\partial t}+\nabla \cdot(\rho U k)=\nabla \cdot\left[\left(\mu+\frac{\mu_{t}}{\sigma_{k}}\right) \nabla k\right]+P_{k}+\rho \varepsilon \\
& \frac{\partial(\rho U)}{\partial t}+\nabla \cdot(\rho U \varepsilon)=\nabla \cdot\left[\left(\mu+\frac{\mu_{t}}{\sigma_{\varepsilon}}\right) \nabla \varepsilon\right]+\frac{\varepsilon}{k}\left(C_{\varepsilon 1} P_{k}-C_{\varepsilon 2} \rho \varepsilon\right) \\
& C_{\varepsilon 1}, C_{\varepsilon 2}, \sigma_{k}, \sigma_{\varepsilon} \text { is constant. }
\end{aligned}
$$

For multiphase, group balance model is used in this paper. Group balance model is based on population balance equations. The population balance equation is as follows:

$$
\frac{\partial}{\partial t} n(m, t)+\frac{\partial}{\partial x^{i}}\left(U^{i}(m, t) n(m, t)\right)=B_{B}-D_{B}+B_{C}-D_{C}
$$

Where $B_{B}, D_{B}, B_{C}, D_{C}$ respectively represent the birth rate due to breakup of larger particles, the death rate due to breakup into smaller particles, the birth rate due to coalescence of smaller particles, and the death rate due to coalescence with other particles.

The group balance composed of different size in the model equation is as follows:

$$
N_{i}(t)=\int_{m_{i-1 / 2}}^{m_{i+1 / 2}} n(m, t) d m
$$




$$
\frac{\partial\left(\rho_{i} r_{i}\right)}{\partial t}+\frac{\partial}{\partial x^{i}}\left(\rho_{i} r_{d} U_{i}^{i}\right)=S_{i}
$$

where $N_{i}$ represent the number density of size group $i, \rho_{i}, r_{i}$ respectively represent density and volume fraction of size group $i, S_{i}$ represents the source term. Breakup models use Luo and Svendsen Model and coalescence models use Prince and Blanch Model [9] [10].

\section{Geometric Modeling and Mesh Generation}

As shown in Fig. 2a, the main features of the gas distributor in slurry column reactor mentioned in this paper are the structure which combined bubble cap structure with preventing-reflux element, FT synthesis reaction in a slurry bubble column reactor belongs to the gas feed. The pressure drop is smaller when the inlet uses a simple conical structure. Meanwhile, the preventing-reflux ball is put into the intake-tube, when working properly and the flow is smooth, while the flow is interrupted supply, the preventing-backflow ball can prevent liquid backflow into the intake-tube. The distributor uses a bubble cap structure with slits, and this special structure on the one hand can expand the diffusion area and refine the bubbles, more importantly, this structure makes gas flow form a baffle at the bottom, which prevents the deposition of catalysts and ensure the security of the whole bed. In this paper, this geometric model is the same experiment size. We use geometries and different meshing strategies to study the aspects of the paper, because the research priorities and methods are different. In this article, ANSYS Workbench provided by Asia-Pacific was used to repair models and mesh geometries. Not all models were used in single gas phase flow calculations, only gas distributor and the lower part of the outer cylinder was used to simplify the calculation of the flow field. In the calculation of the gas-liquid two-phase model, this paper made full use of the symmetry of the geometry, using $1 / 8$ geometry and structured grid. This means not only improved mesh quality but also reduced the number of grid. Therefore, this article ultimately determines the amount of grid was 266,929, and the maximum mesh size was 1mm. As shown in Fig. 2b.

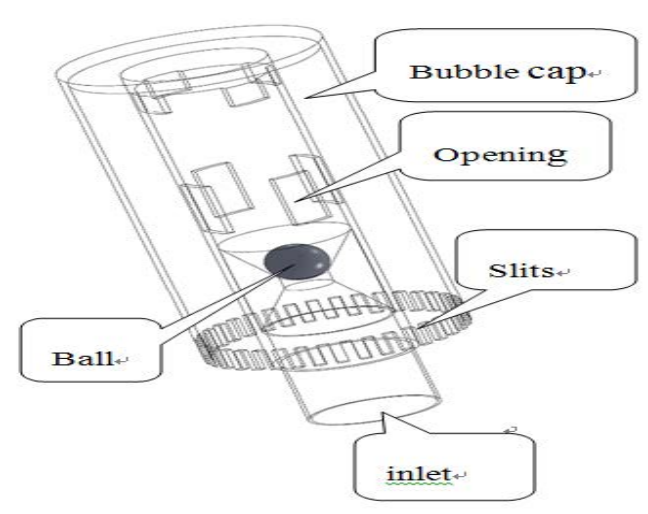

a Gas distributor Structure

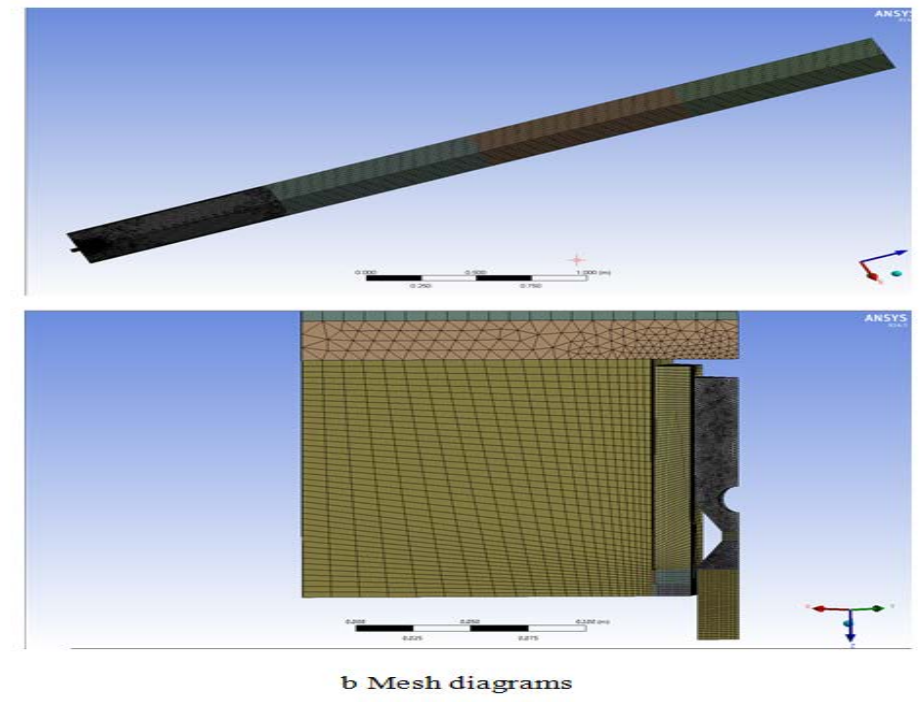

Mesh diagrams

Fig.2. The structure and mesh of bubble column reactor gas distribution

\section{Calculation Results}

The uniformity of the gas distribution, the resistance of flow and the amount of the energy loss will give information for the evaluation of the gas distributor. The unevenness is used to judge the ability of distribution of gas distributor, which is as follows:

$$
M_{f}=\left[\frac{1}{n} \sum_{i=1}^{n}\left(\frac{u_{i}-u}{u}\right)^{2}\right]^{\frac{1}{2}}
$$

$u_{i}$ is local value, $u$ is mean value. It is evident that in an ideal uniform field $\mathrm{M}_{\mathrm{f}}=0$ and the value 
increases with the non-uniformity of distribution.

Fig.3. shows that the gases mostly flow from the upper entrance to the sleeve and the non-uniformity of gas flow will exist when it passes through the small ball. It is interesting to note that there is more gas flow in the upper entrance than in the lower entrance. Part of the gas flow will go in to the sleeve from the lower entrance obliquely, which will generate relative obstruction owing to the gas flow with different flow path and hence cause the chaotic swirl flowing out from the gap. The streamline picture we modeled is exactly identity in this situation. Meanwhile, because of the unsteady and asymmetry of the gas flow in the inner tube, the moving ball shows a chaotic motion which leads to further exacerbation of the chaotic flow in the inner tube.
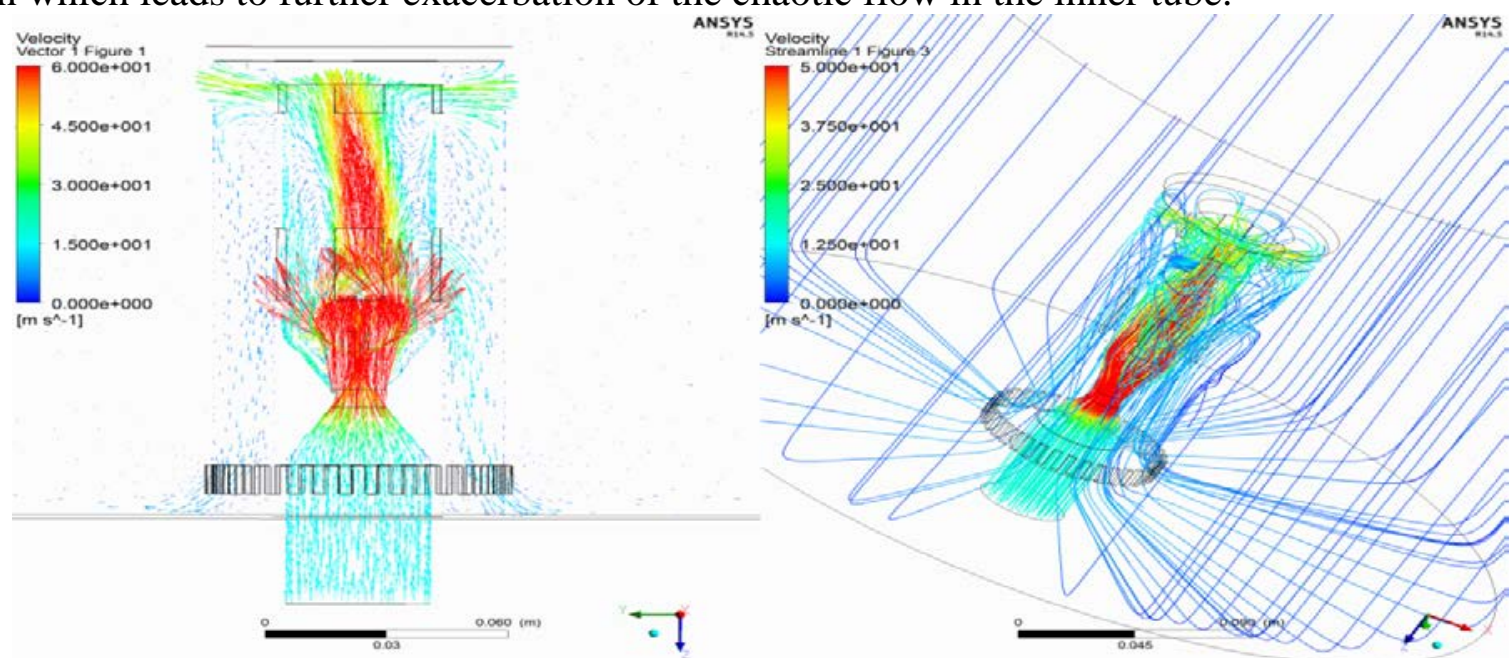

Fig.3. The velocity vectors and streamlines of the flow field

From above analysis, we can see the upper and lower openings cannot be fully utilized and there are blocking air flowing in the opening of annulus. Therefore, the main improvement in this work is to reduce the influence on each mouth for avoiding the stagnation of air flow. This work is mainly focused on two improved structures (Fig.4.), one of which attempt to reduce the relatively closed area via the connection between the upper and lower openings so that can decrease the pressure drop and blockage effectively (Fig.4a.).Another improved structure is utilizing a larger ball because of the irregularity of beating and unsteady of the bed we found in the experiment (Fig.4b.).

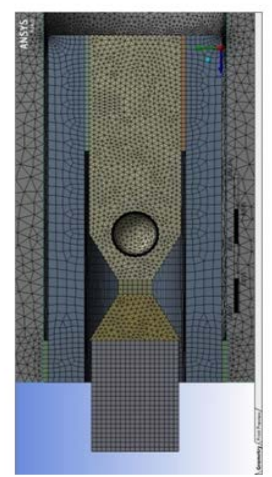

a Improved type 1

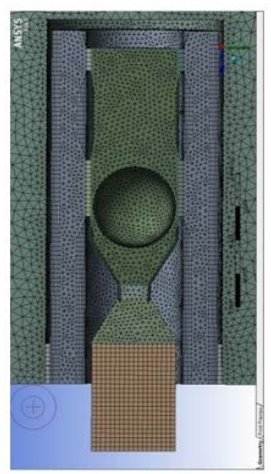

b Improved type 2

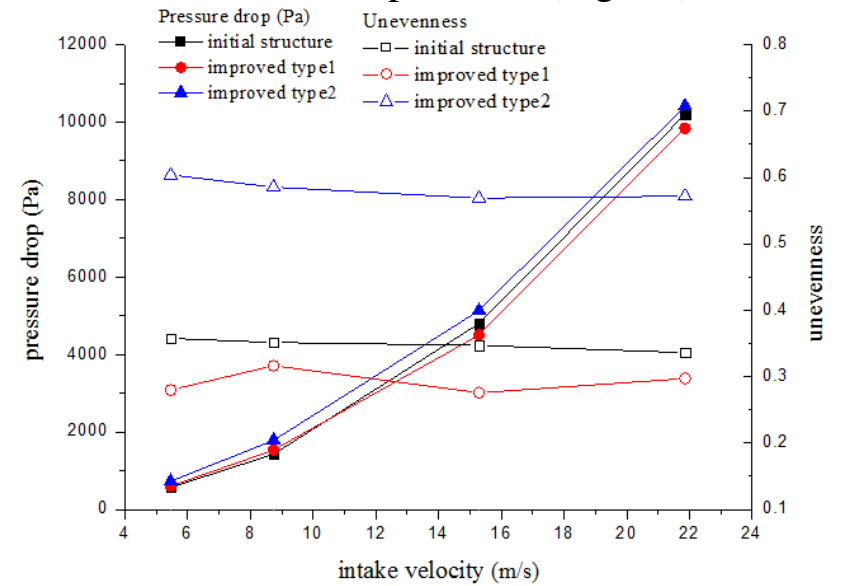

Fig.5. The chart of unevenness and pressure drops

Fig.4. The grids of optimized structures

It is evident that the impact of these three different structures on pressure drop is basically consistent (Fig.5). The combination of upper and lower openings can improve the gas congestion and slightly reduce to the pressure drop. We can note that the pressure drop increase with the ball diameter, which is maybe caused by the dissipation of energy produced via the larger vortex generated by the larger spherical. From the perspective of air flow distribution, the performance of distribution with the combination of upper and lower openings was best in the three types, because it can reduce unevenness by the promotion of turbulence and larger spherical is tend to generate more non-uniform distribution.

The calculation method of the gas holdup is the Bed Expansion Method [11]. As shown in Fig.6 
(a), the overall gas holdup of slurry column reactor increases with increasing working conditions, this trend was consistent with some experiment mentioned by literature [12]. Meanwhile, the overall gas holdup was good in line with the paper's experiment. This proved that the model coupled PBM with Grace Drag Model has a better predictability on bubbling behavior of the slurry column reactor. Fig.6 (b) suggest that most bubbles were large size accounting for $70 \%$ of the total bubbles, small size bubbles was about $20 \%$, and medium bubbles only accounted for around $10 \%$. It could be inferred from the chart that under the illustrated working conditions, bubble sizes were differentiated states, and medium size of air bubbles in this system was more difficult to exist, most bubbles tended to form the larger bubbles. This phenomenon not only provided a reference to the further improvement of gas distributor cap, but also built the foundation for calculating model and selecting particles' size on further optimization.
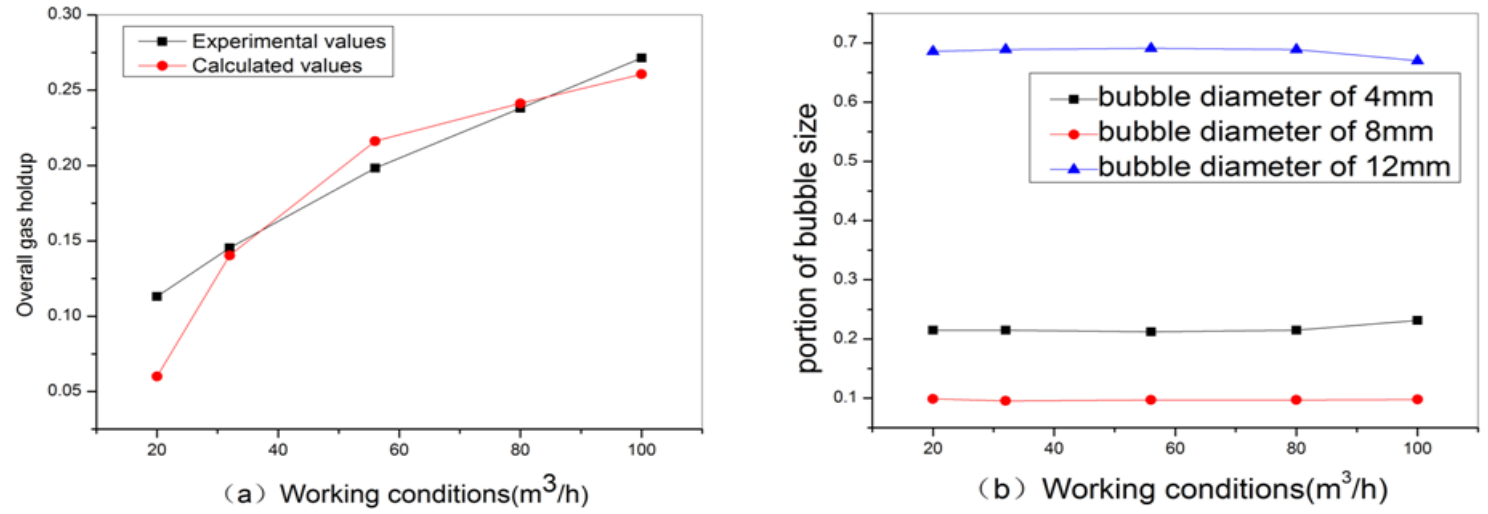

(b) Working conditions $\left(\mathrm{m}^{3} / \mathrm{h}\right)$

Fig.6. The overall gas holdup and bubble size distribution under different working

In the actual industrial production, the reflux of liquid phase and solid phase catalyst often occur because of the disruption or instability generated during the gas supply, and lead to the breakdown of gas distribution nozzle caused by obstruction. Therefore, the research and evaluation of the ability to prevent reflux in distributor is necessary. In order to simplify reflux calculations, we calculated the liquid reflux process under steady bubbling with fixed moving part. Meanwhile, the gas flow through the narrow slit between ball and inner wall with high speed during the bubbling state, which generate a downward force target to ball. We take this force as security margin and calculated free-fall time based on it. Then we compare it with reflux time to evaluate the performance. Fig 7 shows very little liquid go into the funnel area from $0.07 \mathrm{~s}$ and liquid flow out from entrance at $0.08 \mathrm{~s}$. Therefore, the ball secure time should be less than 0.07s. According to the free-fall, we calculated the moving distance during this period time as follows:

$$
s=\frac{1}{2} g t^{2}
$$

By calculating know, the ball should complete the non-return effect after $24.5 \mathrm{~mm}$. Since the difference between the liquid phase reflux under the gas velocity of $5.46 \mathrm{~m} / \mathrm{s}$ and others is negligible, the time taken by liquid phase refluxing from the outer tube to inner tube is very short, about 0.04-0.1s, which put forward higher requirements on the height of entrance and the weight of ball. However, considering the very short reflux time and low liquid flow velocity, the amount of reflux will not be too much. The ball's relatively good non-return ability can meet the demands.

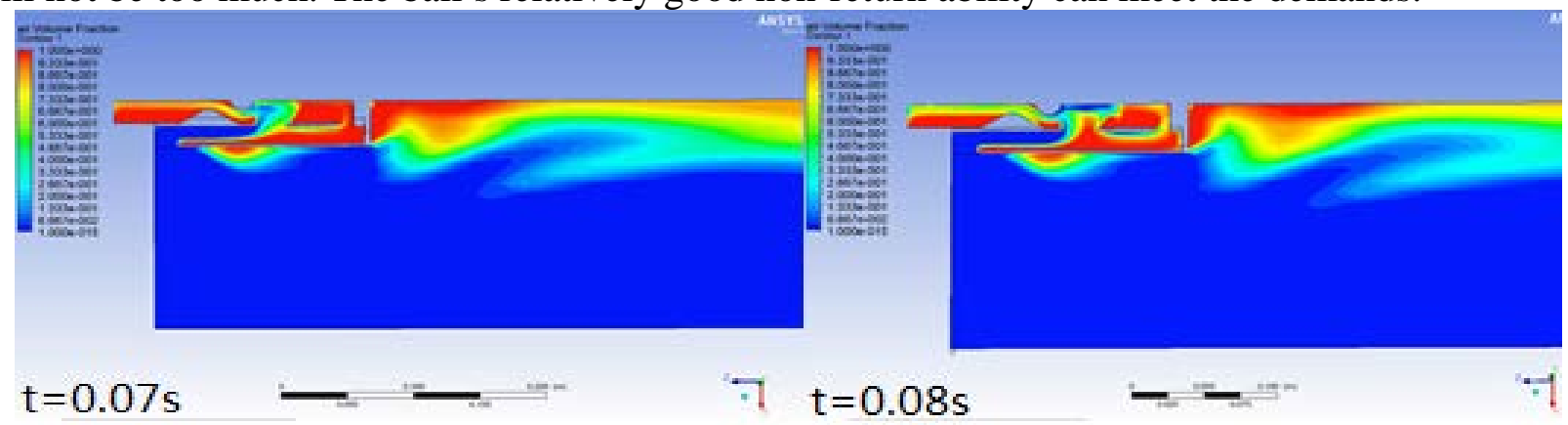

Fig.7. Reflux figure of intake velocity of $5.46 \mathrm{~m} / \mathrm{s}$ 


\section{Conclusion}

The new type gas distributor used in FT synthesis slurry column reactor was numerically studied in the paper. The result showed that the original structure with smaller sphere was beneficial to the reduction of the pressure drop and unevenness. Merging the two openings was helpful to reduce the blocking. The results generated by PBM model and drag force model all can provide excellent condition of bubbles' aggregation and broken, which was mostly identified with the experimental results and literature. In the modeling of preventing reflux, the basic condition was achieved and it takes less than about $0.1 \mathrm{~s}$ for the liquid phase to reflux to the gas region, and hence put up a further requirement for the design of the structure.

\section{References}

[1] Krishna R, Ellenberger J. Gas hold-up in bubble column reactors operating in the chamber and a bubble column, Chemical Engineering Journal. 2007,125(3):149-163.

[2] Ranade V V, Tayalia Y. Modelling of fluid dynamics and mixing in shallow bubble column reactors: influence of sparger design, Chemical engineering Science.2001,56:1667-1675.

[3] Dhotre M T, Ekambara K, Joshi J B. CFD simulation of sparger design and height to diameter ratio on gas hold-up profiles in bubble column reactors, Experimental Thermal and Fluid Science.2004, 28(5):407-421.

[4] Dhotre M T, Joshi J B. Design of a gas distributor: Three-dimensional CFD simulation of a coupled system consisting of a gas chamber and a bubble column, Chemical Engineering Journal, 2007,125(3):149-163.

[5] Krishna R, van Baten J M, Urseanu M I. Three-phase eulerian simulations of bubble column reactors operating in the churn-turbulent regime: a scale up strategy, Chemical Engineering Science, 2000,55(16): 3275-3286.

[6] Dhotre M T, Smith B L. CFD simulation of large-scale bubble plumes: Comparisons against experiments, Chemical Engineering Science, 2007,62(23):6615-6630.

[7] Delnoij E. Kuipers J A M, van Swaaij W P M. Computational fluid dynamics applied to gas-liquid contactors, Chemical Engineering Science, 1997,52(21/22):3623-3638.

[8] Delnoij E, Lammers F A, Kuipers J A M, et al. Dynamic simulation of dispersed gas-liquid two-phase flow using a discrete bubble model, Chemical Engineering Science, 1997,52(9): 1429-1458.

[9] Prince, M. and Blanch, H. , Bubble Coalescence and Break-Up in Air-Sparged Bubble Columns, AIChE Journal 36, 1485-1499.

[10] Luo, S.M., and Svendsen, H., Theoretical Model for Drop and Bubble Breakup in Turbulent Dispersions, AIChE Journal ,42, 1225 -1233.

[11] Songwei, Jingying, Yubo. Review of slurry column reactor, Tianjin Chemical Industry.2009, 23(1):12-14. (in china)

[12] Thorat B N,Shevade A V, Bhilegaonkar K N, Aglawe R H, Veera U P et al. , Effect of Sparger Design and Height to Diameter Ratio on Fractional Gas Hold-up in Bubble Columns, Chemical Engineering Research and Design, 498, 76(7): 823-834. 